\title{
Matrix based Substitution and Diffusion Procedure for Fast Image Encryption
}

\author{
Paul A.J. \\ Caarmel Engineering College \\ Perunad - Ranni \\ Pathanamthitta, Kerala, India
}

\author{
Lekshmi R. Nair \\ Musaliar College of Engineering \\ Malayalapuzha \\ Pathanamthitta, Kerala, India
}

\begin{abstract}
In this paper an encryption technique using matrix based substitution and diffusion for fast image encoding is discussed. The cryptographic procedure manipulates a two dimensional array (matrix) for substitution and diffusion operations. The procedure facilitates fast conversion of plaintext and images into cipher text and cipher images. The paper describes the encryption procedure, discusses on performance and compares with Advanced Encryption Standard (AES). It is shown that the matrix based substitution and diffusion procedure is capable of encrypting images faster than Advanced Encryption Standard.
\end{abstract}

\section{General Terms}

Image encryption, Secure transmission, Information security.

\section{Keywords}

Ciphertext, Decryption, Encryption, Plaintext, Substitution, Diffusion, Secret key, Sub key.

\section{INTRODUCTION}

Protection of data stored in computer hardware as well as transmitted over communication channels is becoming more important [1] in recent times. Text and image encryption are needed for secure transmission of multimedia data using internet communications. Encoding of information in the areas of medical imaging, Tele-medicine and military communications [2] are becoming more significant. Secure communication of images requires encryption at the sending side and decryption at the receiving side. Encryption of large images takes time before they can be transmitted thereby causing considerable delay in successive transmission of encrypted images. In order to minimize the latency, efficient encryption algorithms are needed. An encryption procedure with adequate security and high throughput is sought in multimedia encryption applications. Traditional block ciphers like DES (Data Encryption Standard) [3] and AES (Advanced Encryption Standard) [4] are not efficient when image size is large [5]. Symmetric key cryptography and public key cryptography are available for encryption of information. Public-key algorithms are slow, whereas Symmetric-key algorithms generally run much faster [6]. Symmetric-key cryptography has been (and still is) extensively used to solve the traditional problem of communication over an insecure channel [7]. High throughput encryption and decryption are becoming increasingly important in the area of high-speed networking $[8,9]$. Fast encryption algorithms are needed these days for high-speed secure communication of multimedia data [10]. A fast symmetric key encryption procedure, Matrix Array symmetric Key Encryption (MASK) for efficient image encryption has been proposed in [11]. In this paper, substitution and diffusion operations based on matrix manipulations for image encryption is presented. The substitution and diffusion procedure presented here is in the form of a block cipher with a block size of 128 bits and key size of 128 bits. Rest of the paper is organized in the following sections. In section 2, substitution and diffusion procedure is explained and in section 3, Performance of the procedure is compared with Advanced Encryption Standard. A basic security analysis, through statistical approach, is offered in section 4 and conclusions are made in section 5 .

\section{SUBSTITUTION AND DIFFUSION}

The substitution and diffusion procedure consists of two basic steps. The first step involves initialization of a matrix, $M$ using secret key $K$ and the generation of two matrices of sub keys for iterative substitution and diffusion round operations. The second step involves substitution and diffusion operations in iterations. Figure 1 shows the simplified block diagram of substitution and diffusion procedure.

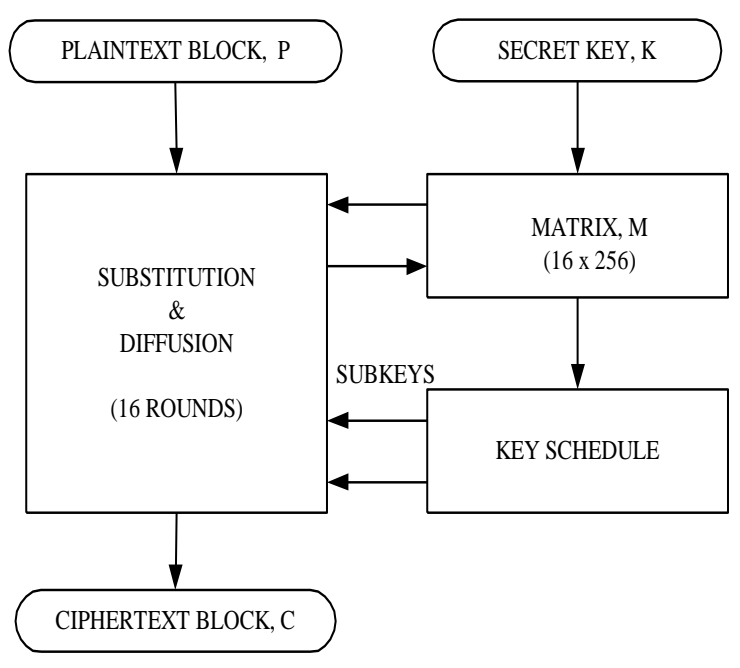

Figure 1: Simplified block diagram of substitution and diffusion procedure

\subsection{Matrix Initialization}

A matrix $M$ with 16 rows and 256 columns is defined. Each column of every row is filled with a number between 0 and 255 (both the numbers included) in an order depending on the characters of secret key. The first column in the $\mathrm{i}^{\text {th }}$ row of the matrix is filled with integer value of $i^{\text {th }}$ character of the secret key, $K$ (that is, $M[i][1]=$ Integer value of $K[i]$ ). The subsequent columns of the $i^{\text {th }}$ row of the matrix are filled with numbers that have increments of 1 from the previous column value till the number is 255 . Subsequent columns are filled with numbers starting from 0 and ending with integer value of the $i^{\text {th }}$ character of secret key minus 1 . The 
distribution of characters in the columns of all the sixteen rows of the matrix thus becomes key dependent. Without knowing the secret key the element in a column of any row of the matrix $M$ cannot be determined by an adversary. Plate 1 shows the matrix initialization pseudo code.

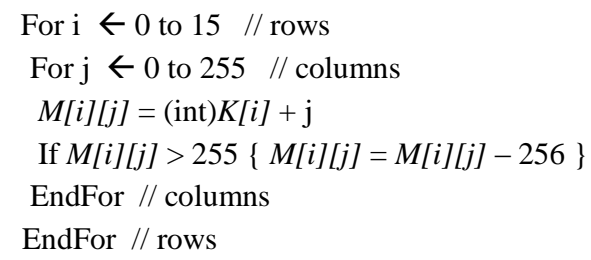

\section{Plate 1: Matrix initialization pseudo code}

\subsection{Key Schedule}

Sub-keys used in round operations are generated by key scheduling procedure. In this procedure two sub-key matrices $K s_{I}$ and $K s_{2}$ (of size $16 \times 16$ ) are derived from the base matrix $M$. These pairs of key can be used in substitution and diffusion operations performed in a typical block cipher. It is desirable that the key scheduling be a complex procedure so that an adversary must find it extremely difficult to derive the sub-keys during crypt analysis [12]. Another desirable feature of key schedule is that a small change in the secret key should get well diffused in to the sub-keys. This means that one bit change in secret key should cause many bits to change in subkeys. These two desirable features are considered while designing the key scheduling procedure. The procedure is explained in steps as follows:

1) Secret key, K, is transposed (T) to get Ka1. It is a bytelevel transposing operation where by the LS byte takes the place of MS byte position and the MS byte takes the LS byte position after the transpose operation. For example, if, bytes in array, $\mathrm{K}$, is $\{\mathrm{K} 0, \mathrm{~K} 1, \mathrm{~K} 2, \mathrm{~K} 3, \ldots . \mathrm{K} 14, \mathrm{~K} 15\}$ then, after performing the transpose operation, $\mathrm{Ka} 1=\mathrm{K}$ Transposed, the contents of Ka1 will become $\{\mathrm{K} 15$, $\mathrm{K} 14, \ldots . \mathrm{K} 5, \mathrm{~K} 4, \mathrm{~K} 3, \mathrm{~K} 2, \mathrm{~K} 1, \mathrm{~K} 0\}$.

2) $K a l$ is XOR ed with $K$ to get $K a 2$. This operation can cause up to 2 bits to change in $K a 2$ when 1 bit is changed in secret key $K$

3) Left half of $\mathrm{Ka} 2$ and right half of $\mathrm{Ka} 2$ is $\mathrm{XOR}$ ed to get $\mathrm{Ka} 3$.

4) Transposed left half of $K a 2$ and transposed right half of $\mathrm{Ka} 2$ are XOR ed to get $\mathrm{Ka} 4$.

5) $\mathrm{Ka} 3$ and $\mathrm{Ka} 4$ are concatenated to get Ka5. With this operation, 1 bit change in secret key, $\mathrm{K}$, can cause up to 4 bits to change in Ka5.

6) Sum of integer values of bytes in Ka5 is calculated to get $\mathrm{L}$

7) Kse1 is calculated such that Kse1 = L \% 23.

When secret key has 1 bit change, Kse1 can have up to 4 counts change.

8) Kse2 is calculated such that Kse2 $=\mathrm{L} \% 15$.

When secret key has 1 bit change, Kse2 can have up to 4 counts change and (Kse1 + Kse2) can have up to 8 counts change.

9) Two matrices Ks1 and Ks2 of size $16 \times 16$ are derived from the base matrix, $\mathrm{M}$, such that
Ks1[row][column] $=\mathrm{M}[$ row] $[$ Kse1+Kse2+column $]$ Ks2[row][column]=M[row][Ks1[row][column]]

Columns of Ks1 matrix are chosen from the base matrix M depending upon Kse1 and Kse2 Values. Here, an element of Ks1 can have up to 8 count change with one bit change in secret key.

Columns of Ks2 matrix are chosen from the base matrix M depending upon element values of columns of Ks1 matrix. An element of Ks2 can have up to 8 counts change with one bit change in secret key.

10) Rotate vertically down $\mathrm{i}^{\text {th }}$ column of matrix $K s I$ number of times equal to $((\operatorname{int}(K[i]) \% 12)+K s e 1)$.

11) Rotate vertically down $i^{\text {th }}$ column of matrix Ks 2 number of times equal to $((\operatorname{int}(K[i]) \% 10)+K s e 2)$. The vertical rotations shuffle the elements of sub-key matrices thereby providing more changes in the sub-key values while one bit change is applied on the original secret key, $K$. Plate 2 shows the pseudo code of key schedule procedure.

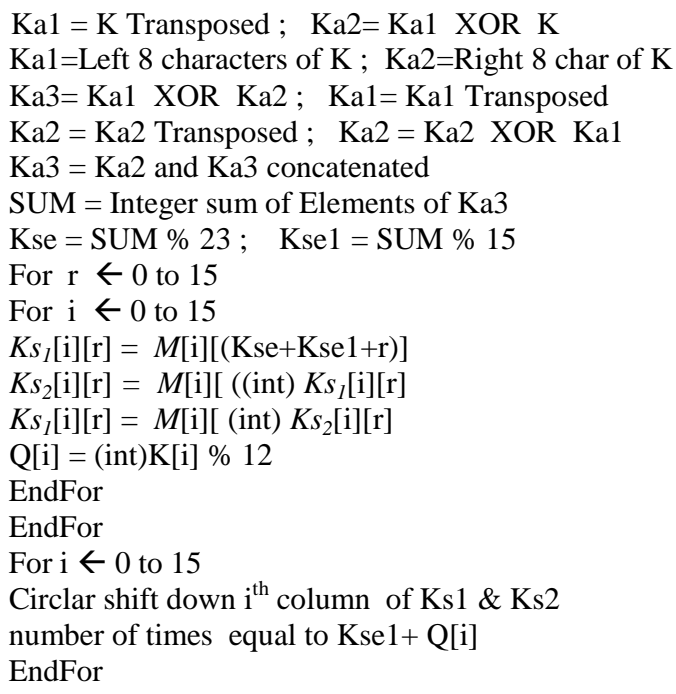

Plate 2: Key schedule pseudo code

\subsection{Substitution and Diffusion Iterations}

There are up to16 iterations of substitution and diffusion in the cipher. A data block, $P$, consisting of 16 bytes, $P(0) \rightarrow$ $P(15)$, is applied at the input of substitution and diffusion iterative round unit. Data byte, $P(i)$ is taken and the decimal value of $P(i)$ is used as column number, $\mathrm{j}$, of the $\mathrm{i}^{\text {th }}$ row of matrix $M$ to read the value $M(i, j)$. This value is taken as the substitute for $P(i)$. For example, for the byte $P(0)$ in a block, i $=0$ and $\mathrm{j}=$ decimal value of $P(0)$ are used to find the value $M(0, j)$ as substitute, $C(0)$, for $P(0)$. In this way, all the 16 bytes of data in a block are substituted by a value taken from selected column and row of the matrix depending on position of the data in the block and the data value. The diffusion of data is facilitated by key based XOR, data based XOR, transpose (T) and data based rotation ( $\gg>$ ) operations as given in the pseudo code. The output of last iteration round is taken as the ciphertext block. Number of iterations can be chosen between 1 and 16. Plate 3 shows Substitution and diffusion pseudo code. 
For $\mathrm{i} \leftarrow 0$ to 15

$\mathrm{j}=\mathrm{P}(\mathrm{i}))$

$\mathrm{C}(\mathrm{i})=\mathrm{M}[\mathrm{i}][\mathrm{j}]$

EndFor

$\mathrm{C}=\mathrm{C}$ EXOR Ks1

$\mathrm{C}=\mathrm{C}$ Transpose

$\mathrm{C} 1=$ left half bytes of $\mathrm{C}$

$\mathrm{C} 2=$ right half bytes of $\mathrm{C}$

$\mathrm{C} 2=\mathrm{C} 1$ EXOR $\mathrm{C} 2 ; \quad \mathrm{C} 1=\mathrm{C} 1$ Transpose

$\mathrm{C} 1=\mathrm{C} 1 \mathrm{EXOR} \mathrm{C} 2$

$\mathrm{C} 3=\mathrm{C} 1 \| \mathrm{C} 2 ; \mathrm{C} 3=\mathrm{C} 3$ Transpose

$\mathrm{C} 3=\mathrm{C} 3 \mathrm{EXOR} \mathrm{Ks} 2$

$\mathrm{C} 4=$ Right half bytes of $\mathrm{C} 3$

$\mathrm{C} 5=$ left half bytes of $\mathrm{C} 3$

C6=byte sum C5

C6=C6 MODE 6

Rotate right $\mathrm{C} 4$, $\mathrm{C} 6$ times

$\mathrm{C} 7=$ byte sum $\mathrm{C} 4 ; \mathrm{C} 7=\mathrm{C} 7 \mathrm{MODE} 6$

Rotate right $\mathrm{C} 5, \mathrm{C} 7$ times; $\mathrm{C}=\mathrm{C} 4 \| \mathrm{C} 5$

$\mathrm{P}=\mathrm{C}$

Plate 3: Substitution and diffusion pseudo code

\section{Performance Analysis}

The encryption algorithm has been tested with different images of various sizes and the results are compared with AES. The simulation tests have been conducted using Matlab7 in an Intel Atom $1600 \mathrm{MHz}$ processor with Windows-xp operating system. Measurement of encryption speed, encryption quality and key sensitivity tests have been made. Figure 2 shows encryption and decryption of an image of size $128 \times 128$ pixels, by the proposed MASK encryption and AES encryption.

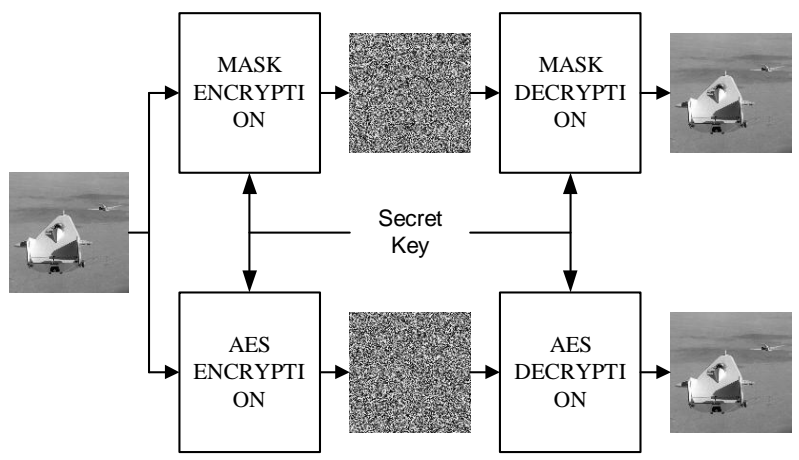

Figure 2: Encryption and decryption of image by MASK and AES

\subsection{Measurement of Encryption Quality}

The encryption quality, $Q_{e}$, may be expressed in terms of total changes in pixel gray values between the original image and the encrypted image given by:

$$
\mathrm{Q}_{\mathrm{e}}=\frac{\sum_{L=0}^{255}\left|\mathrm{H}_{\mathrm{L}}(\mathrm{F})-\mathrm{H}_{\mathrm{L}}\left(\mathrm{F}^{\prime}\right)\right|}{256}
$$

Where, $L$ is the pixel gray level, $H_{L}(F)$ the number of pixels having gray level $\mathrm{L}$ in the original image and $\mathrm{H}_{\mathrm{L}}\left(\mathrm{F}^{\prime}\right)$ the number of pixels having gray level $\mathrm{L}$ in the encrypted image. The encryption quality has been measured using different images of same size as well as different sizes. Figure 3 shows comparison of encryption quality measured in AES and
MASK with different images of size $512 \times 512$ pixels. Figure 4 shows comparison of encryption quality measured in AES $\&$ MASK with same image of sizes (a) $128 \times 128$, (b) $256 \times$ 256 and (c) $512 \times 512$ pixels. It could be seen that the encryption quality in both MASK and AES algorithms are more or less same.

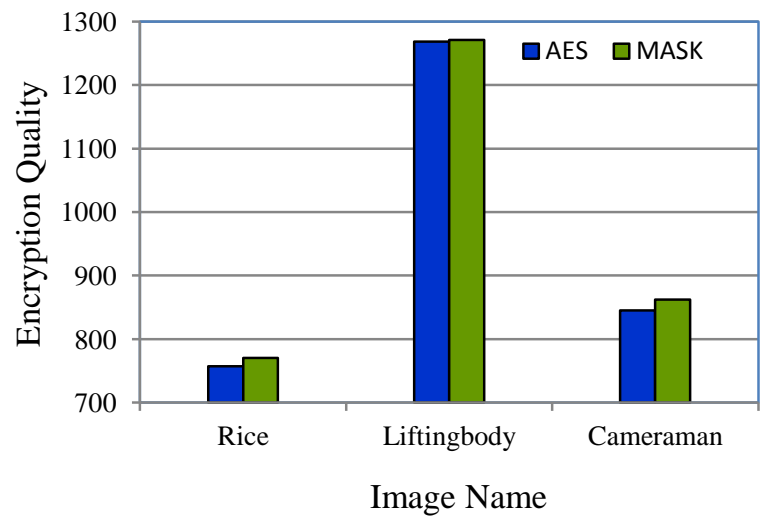

Figure 3: Encryption quality with different images of same size

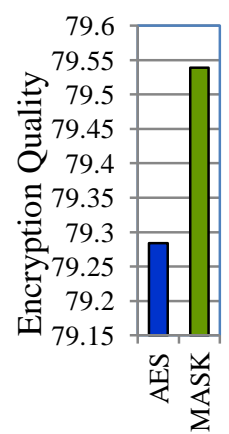

(a)

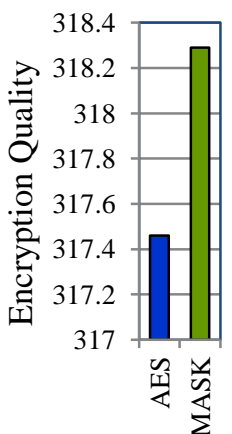

(b)

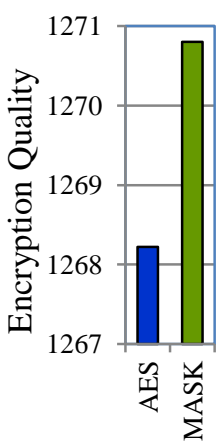

(c)
Figure 4: Encryption quality with same image of different sizes

\subsection{Measurement of Encryption Speed}

Encryption speed of the encryption scheme has been measured and compared with AES. Three images of size $128 \times 128,256 \times 256$ and $512 \times 512$ were chosen for the measurement. Figure 5 shows the average encryption speeds observed using these images for different rounds in AES and MASK.

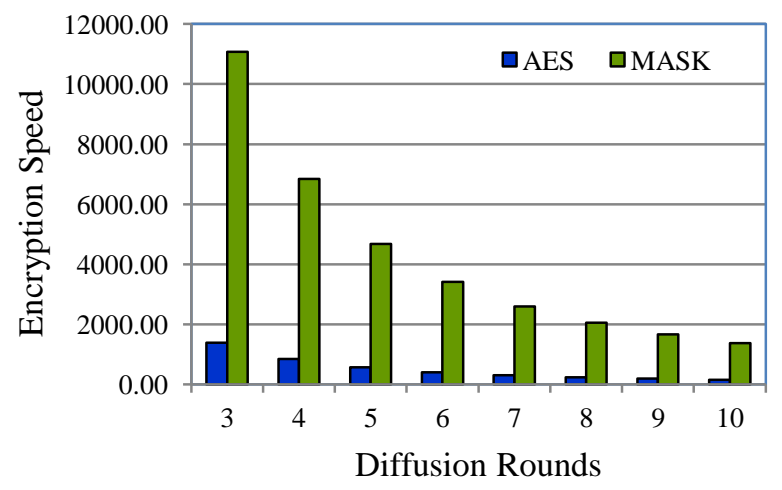

Figure 5: Encryption speed vs. diffusion rounds 
Figure 6 shows the encryption speeds observed using three different images for same size $(256 \times 256$ pixels $)$ in AES and MASK. The average encryption time and decryption time is used to compute the speed in bytes / second.

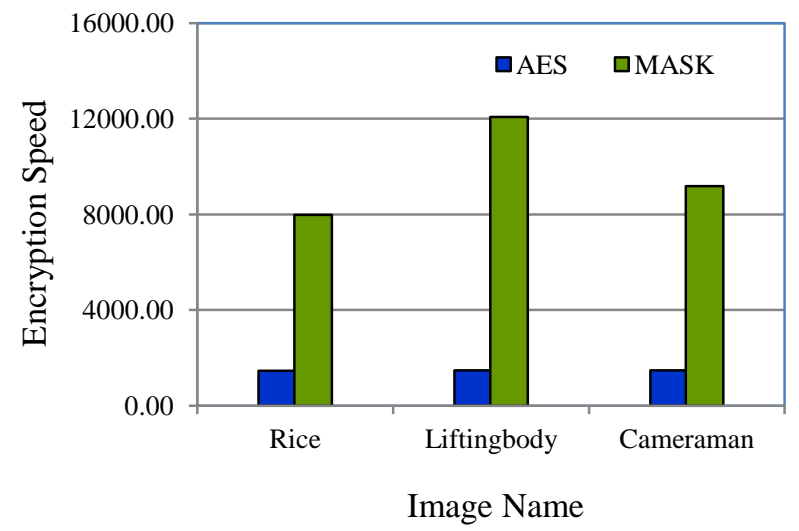

Figure 6: Average encryption speed with different images of same size

\subsection{Key Space Analysis}

A block cipher should be sensitive to the secret keys and the key space should be large enough to resist ciphertext-only (brute-force) attack. The MASK cipher use 128 bits key size that provides $2^{128}$ key combinations.

\subsubsection{Exhaustive Key Search}

An adversary may try to directly guess the secret key $K$. Although $K$ is user specified, it can be generated as a random number or a set of random numbers each independent of the other. The key size is defined as 128 bits in the proposed algorithm. This means that $K$ has $2^{128}$ possible combinations. If an adversary employs a 100 MIPS computer to find $K$, the computational load is $2^{128} /\left[\left(100 * 10^{6}\right) * 60 * 60 * 24 * 365\right]$ or over $10^{23}$ years. Like any other cipher having 128 bit secret key, the MASK cipher is resistant to ciphertext-only attack.

\subsubsection{Key Sensitivity Test}

In this test we first run the encryption program, MASK, with an input image, $I$, and a secret key, $K$, and obtain the cipher image, $C 1$. Then we run the program with the same input image and another secret key that is different by one bit (closest key) with respect to the first key and obtain the cipher image, $C 2$. Using the two encrypted images we obtain the difference image, /(C1-C2)/.

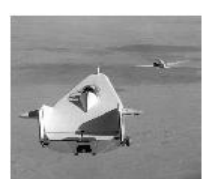

(a)

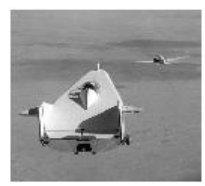

(e)

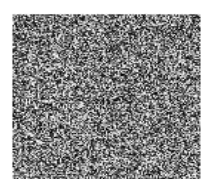

(b)

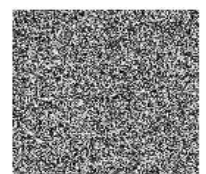

(f)

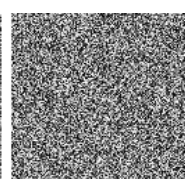

(c)

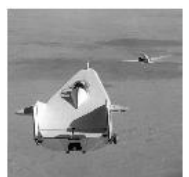

(g)

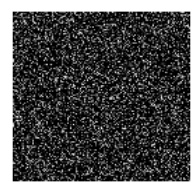

(d)

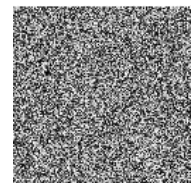

(h)
Figure 7: Encryption and decryption results using closest keys
It has been observed that the image encrypted by the first key has $34.57 \%$ of difference from the image encrypted by the second key in terms of pixel gray scale values although there is only one bit difference in the two keys. Figure 7(a) shows the input image of "Lifting body", (b) shows the encryption using the first key, (c) shows encryption using the closest key and (d) shows the difference image. Decryption test is also done with the two closest keys and results were obtained. Figure 7(e) shows the input image of "Lifting body", 7(f) shows the encryption using the first key, 7(g) shows decryption using the first key and 7(h) shows the decryption using the second key which is closest to the first key. It may be noted here that the decrypted image with the closest key does not reveal any information contained in the original image.

\section{SECURITY ANALYSIS}

An encryption scheme must be able to resist all known attacks such as known-plaintext attack, ciphertext attack, statistical attack, differential attack and various brute force attacks. Some security analysis performed on the proposed encryption scheme includes statistical analysis and key space analysis. It can be seen that in all the analysis performed, the results obtained from MASK encryption compares well with the results obtained from AES encryption. The methods of confusion and diffusion are used to frustrate powerful attacks based on statistical analysis [9]. The proposed encryption scheme, MASK, demonstrates confusion and diffusion that can stand against statistical attacks. This is shown by histograms of ciphered images and adjacent pixel autocorrelation plots. It can be seen that in all the analysis performed, the results obtained from MASK encryption is compares well with the results obtained from AES encryption.

\subsection{Histogram Analysis of Cipher Images}

Black \& white images of different sizes have been used to obtain the histograms of encrypted images using the proposed encryption scheme and AES. It has been observed that the histograms of encrypted images have fairly uniform distribution of pixel gray values and significantly different from the histogram of the original image. Figure 8 (a), (b) and (c) shows test image "Lena", encrypted images obtained from MASK, and AES. Their respective histograms are shown in figure 8(d), (e) and (f).

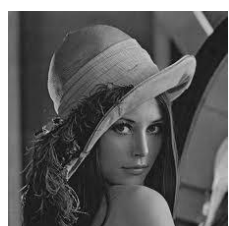

(a)

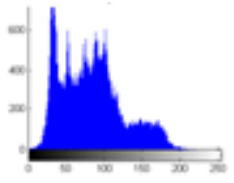

(d)

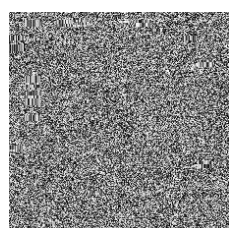

(b)

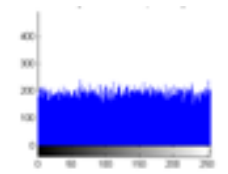

(e)

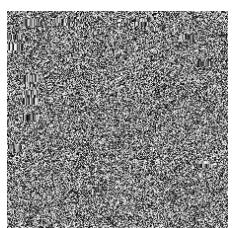

(c)

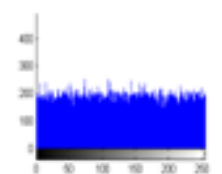

(f)
Figure 8: Image "Lena", cipher images and histograms

It may be noted that the histograms of encrypted images exhibit fairly uniform gray level distribution suggesting strong diffusion of pixel gray values.

\subsection{Adjacent Pixel Correlation Analysis}

A photographic image usually will have strong correlation 
between adjacent picture elements. This property enables the rough prediction of the pixel values surrounding any picture element. In a well encrypted image this should not be the case because an adversary should not be able to decode the encrypted image using this property. Thus in a well encrypted image, it is ensured that the adjacent pixels will exhibit very weak correlation. Correlation between two adjacent pixels along horizontal, vertical and diagonal directions has been obtained using the original images and the ciphered images. Two adjacent vertical lines, horizontal lines and diagonal lines have been selected from the plain images and the ciphered images to obtain the correlation plot. Figure 9(a) shows adjacent pixel correlation plots, in horizontal, vertical and diagonal directions of image "Onion" used for encryption by MASK \& AES. Figure 9(b) shows adjacent pixel correlation plots, in horizontal, vertical and diagonal directions in the cipher image by MASK. Figure 9(c) shows adjacent pixel correlation plots, in horizontal, vertical and diagonal directions in the cipher image by AES. The horizontal and vertical axes represent pixel gray levels in selected pairs of adjacent pixels. It can be seen that in the correlation plots of the encrypted images by MASK and AES the correlation is very low in all the three directions as the pixel values are distributed in random. This indicates that both encryptions produce uncorrelated adjacent output bytes from correlated input adjacent bytes.
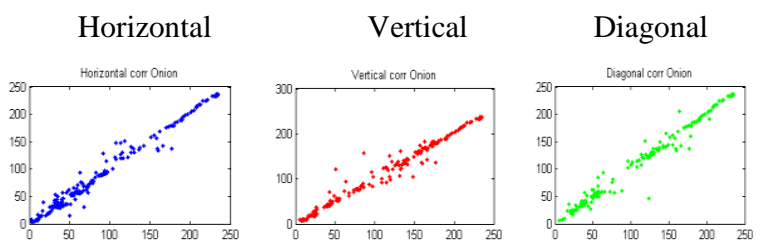

(a) Adjacent pixel correlation plots of image "Onion"
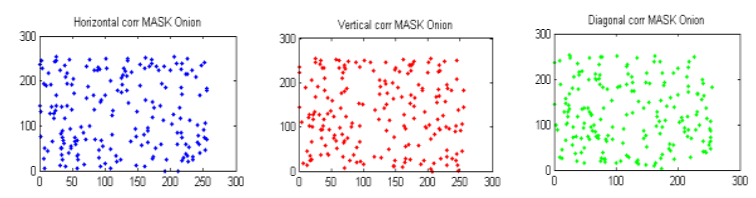

(b) Adjacent pixel correlation plots of cipher image (MASK)
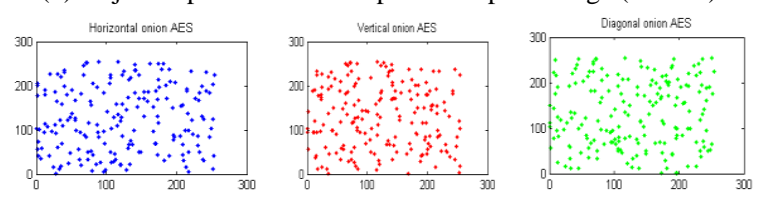

(c) Adjacent pixel correlation plots of cipher image (AES)

\section{Figure 9: Adjacent pixel correlations of original image “Onion", MASK and AES encrypted images}

\section{CONCLUSION}

We have presented a new cryptographic procedure, using matrix based substitution and diffusion operations. The matrix-based substitution facilitates poly-alphabetic nonlinear substitution. Multiple round operations depending on secret key and data values give adequate diffusion of information values. The security of the algorithm is comparable with that of AES as indicated by encrypted images, their histograms and correlation plots. A basic security analysis has been made based on histograms of encrypted images and correlation data. The performance test results indicate the suitability of MASK for fast image encryption. It has been shown that MASK encryption is eight folds faster than AES. Additional test and analysis of the algorithm can be conducted to find the suitability of the algorithm for audio and video encryption.

\section{REFERENCES}

[1] Ju Young O.H et-al, "A Selective Encryption Algorithm based on AES for Medical Information," Health Informatic Research, Vol 16, No. 1, March 2010, pp. 2229.

[2] M.Zeghid et-al, "A Modified AES based Algorithm for Image Encryption," World Academy of Science, Engineering and Technology, 27 2007, pp 206 - 210.

[3] Data Encryption Standard : http://csrc.nist.gov/publications/fips/fips 46-3/fips- 463.pdf

[4] Advanced Encryption Standard: http://csrc.nist.gov/publications/fips/fips197/ fips197.pdf

[5] Xiaogang Jia et-al, "Image Encryption using IKEDA Map,"

International Conference on Intelligent Computing and Cognitive Informatics, IEEE Computer Society, pp. 455$458,(2010)$

[6] Jose J. Amador, Robert W. Green, "Symmetric-Key Block Ciphers for Image and Text Cryptography," International Journal of Imaging System Technology, Vol. 15 - pp. 178-188, (2005).

[7] Dragos Trinca, "Sequential and Parallel Cascaded Convolution Encryption with Local Propagation: Toward Future Directions in Cryptography," Proceedings of The third International Conference on Information Technology-New Generations. (ITNG'06), 0-7695-24974 / 2006, IEEE Computer Society, (2006).

[8] Adam J. Elbirt, Christof Paar “An Instruction- Level Distributed Processor for Symmetric-Key Cryptography," IEEE Transactions on Parallel and distributed Systems, Vol. 16, No. 5, May, (2005).

[9] Paul A.J., Varghese Paul, P. Mythili "Fast Symmetric Cryptography using Key and Data based Masking Operations," International Journal of Computational Intelligence-Research and Applications. Vol. 3, No. 1, January-June 2009, pp 5-10.

[10] Krishnamoorthy G.N, V. Ramaswamy, "Encryption Quality Analysis and Security Evaluation of CAST-128 Algorithm and its Modified Version using Digital Images," International Journal of Network Security \& its Applications, Vol.1, No.1, April 2009, pp 28-33.

[11] Paul A.J., P. Mythili, K. Poulose Jacob, "Matrix based Cryptographic procedure for Efficient Image encryption", Proceedings of International Conference on Recent Advances in Intelligent Computational Systems, RAICS IEEE, Sept - 2011, pp. 173-177.

[12] Paul A.J., Saju A., Lekshmi R. Nair, "Data based Transposition to Enhance Data Avalanche and Differential Data Propagation in Advanced Encryption Standard". International Journal of Computer Applications vol. 67 No. 12, April 2013, pp. 6-9. 